\title{
Quantum corrections to the thermodynamics of Schwarzschild-Tangherlini black hole and the generalized uncertainty principle
}

\author{
Z. W. Feng ${ }^{1, \mathrm{a}}$, H. L. Li ${ }^{1,2}$, X. T. Zu ${ }^{1, \mathrm{~b}}$, S. Z. Yang ${ }^{3, \mathrm{c}}$ \\ ${ }^{1}$ School of Physical Electronics, University of Electronic Science and Technology of China, Chengdu 610054, China \\ ${ }^{2}$ College of Physics Science and Technology, Shenyang Normal University, Shenyang 110034, China \\ ${ }^{3}$ Physics and Space Science College, China West Normal University, Nanchong 637009, China
}

Received: 31 December 2015 / Accepted: 31 March 2016 / Published online: 16 April 2016

(C) The Author(s) 2016. This article is published with open access at Springerlink.com

\begin{abstract}
We investigate the thermodynamics of Schwarzschild-Tangherlini black hole in the context of the generalized uncertainty principle (GUP). The corrections to the Hawking temperature, entropy and the heat capacity are obtained via the modified Hamilton-Jacobi equation. These modifications show that the GUP changes the evolution of the Schwarzschild-Tangherlini black hole. Specially, the GUP effect becomes susceptible when the radius or mass of the black hole approaches the order of Planck scale, it stops radiating and leads to a black hole remnant. Meanwhile, the Planck scale remnant can be confirmed through the analysis of the heat capacity. Those phenomena imply that the GUP may give a way to solve the information paradox. Besides, we also investigate the possibilities to observe the black hole at the Large Hadron Collider (LHC), and the results demonstrate that the black hole cannot be produced in the recent LHC.
\end{abstract}

\section{Introduction}

One common feature among various quantum gravity theories, such as string theory, loop quantum gravity, and noncommutative geometry, is the existence of a minimum measurable length which can be identified with the order of the Planck scale [1-4]. This view is also advocated by many Gedanken experiments [5]. The minimum measurable length is especially important since it can be applied to different physical systems and modify many classical theories [6-13]. One of the most interesting modified theories is called the generalized uncertainty principle (GUP), which is a gener-

\footnotetext{
a e-mail: zwfengphy@163.com

be-mail: xtzu@uestc.edu.cn

c e-mail: szyangxwnu@126.com
}

alization of the conventional Heisenberg uncertainty principle (HUP). It is well known that the uncertainty principle is closely related to the fundamental commutation relation. Therefore, taking account of the minimum measurable scale, Kempf, Mangano and Mann proposed a modified fundamental commutation relation

$\left[x_{i}, p_{j}\right]=i \hbar \delta_{i j}\left[1+\beta p^{2}\right]$,

with the position and momentum operators

$x_{i}=x_{0 i}, \quad p_{j}=p_{0 j}\left(1+\beta p_{0}^{2}\right)$,

where $x_{0 i}$ and $p_{0 j}$ satisfy the canonical commutation relations $\left[x_{0 i}, p_{0 j}\right]=i \hbar \delta_{i j}[14]$. Through the above equations, the most studied form of the GUP is derived as

$\Delta x \Delta p \geq \frac{\hbar}{2}\left[1+\beta(\Delta p)^{2}\right]$,

where $\Delta x$ and $\Delta p$ represent the uncertainties for position and momentum. The $\beta=\beta_{0} \ell_{p}^{2} / \hbar^{2}=\beta_{0} / M_{p}^{2} c^{2}, \beta_{0}\left(\leq 10^{34}\right)$ is a dimensionless constant, and $\ell_{p}$ and $M_{p}$ are the Planck length $\left(\sim 10^{-35} \mathrm{~m}\right)$ and Planck mass, respectively. In the HUP framework, the position uncertainty can be measured to an arbitrary small value since there is no restriction on the measurement precision of the momentum of the particles. However, Eq. (3) implies the GUP existence of a minimum measurable length $\Delta x_{\min } \approx \ell_{p} \sqrt{\beta_{0}}$. In the limit $\Delta x \gg \ell_{p}$, one recovers the HUP $\Delta x \Delta p \geq \hbar / 2$.

The implications of the aspects of GUP have been investigated in many contexts such as modifications of the quantum Hall effect [15], neutrino oscillations [16], Landau levels [17], cosmology $[18,19]$, the weak equivalence principle (WEP) [20], and Newton's law $[21,22]$. It should be noted 
that the GUP has also influence on the thermodynamics of black holes. In an elegant paper, Adler, Chen and Santiago proposed that the $\Delta p$ and $\Delta x$ of the GUP can be identified as the temperature and radius of the black hole. With this heuristic method (the Hawking temperature-uncertainty relation), the GUP's impacts on the thermodynamics of Schwarzschild (SC) black hole have been discussed in [23]. This work showed that the modified Hawking temperature is higher than the original case, and the GUP effect leads to remnants in the final stages of black hole evaporation. This interesting work has widely got attention, many other black holes' thermodynamics features have been studied with the help of the Hawking temperature-uncertainty relation [24-28].

On the other hand, the thermodynamics of black holes also can be calculated by the tunneling method [29-34]. The tunneling method was first proposed by Parikh and Wilczek for investigating the tunneling behaviors of massless scalar particles [29]. Later, this method was extended to a study of the tunneling of massive and charged scalar particles [30]. The Hamilton-Jacobi ansatz is another kind of tunneling method [31-33]. With the help of the Hamilton-Jacobi ansatz, Kerner and Mann have carefully analyzed fermion tunneling from black holes [34]. So far, the tunneling method plays an important role in studying black hole radiation, it can effectively help people further understand the properties of black holes, gravity, and quantum gravity [35-39].

Combining the GUP with the tunneling method, Nozari and Mehdipour studied the modified tunneling rate of the SC black hole [40]. Subsequently, many more papers on the subject appeared, aiming to investigate the GUP corrected temperature of complicated spacetimes [41-47]. However, as far as we know, those works are limited to low dimensional spacetimes. It is well known that the higher dimensional spacetimes include more physics information, moreover, one of the most exciting signatures is that people may detect the black holes in the large extra dimensions by using the Large Hadron Collider (LHC) and Ultrahigh Energy Cosmic Ray Air Showers (UECRAS) [48-54]. In other words, the large extra dimensions have opened up new doors of research in black holes and quantum gravity. Therefore, in this paper, we will investigate the GUP corrected thermodynamics of Schwarzschild-Tangherlini (ST) black hole by using the quantum tunneling method. The ST black hole is a typical higher dimensional black hole, people can get many new solutions of higher dimensional spacetimes via the ST metrics. In [55], the authors showed that the ST black hole is a good approximation to a compactified spacetime when the compact dimension's size is much larger than the black hole's size. Thus, the ST black hole is a good tool for researching the distorted compactified spacetime. Based on the above arguments, we think the GUP corrected thermodynamics of the ST black hole is worth to be studied. By utilizing the tunneling method and the GUP, we find that the modified temperature is lower than the original case. Meanwhile, it is also in contrast to the earlier findings, which are analyzed by the Hawking temperature-uncertainty relation $[23,51]$. When the mass of the ST black hole reaches the order of the Planck scale, the GUP corrected thermodynamics decreases to zero. This in turn prevents the black hole from evaporating completely and leads to a remnant of the ST black hole.

The outline of the paper is as follows. In Sect. 2, incorporating GUP, we derive the modified Hamilton-Jacobi equations in curved spacetime via the WKB approximation. In Sect. 3, the tunneling radiation of particles from the ST black hole is addressed. In Sect. 4, due to the GUP corrected temperature, we analyze the remnants of ST black hole. In Sect. 5 , we investigate the minimum black hole energy to form a black hole in the LHC. The last section is devoted to our conclusion.

\section{Modified Hamilton-Jacobi equations}

In this section, we will derive the modified Hamilton-Jacobi equations from the generalized Klein-Gordon equation and the generalized Dirac equation. Based on the momentum operators of Eq. (2), the square of the momentum takes the form $[42,43]$

$p^{2}=p_{i} p^{i} \simeq-\hbar^{2}\left[1-2 \beta \hbar^{2}\left(\partial_{j} \partial^{j}\right)\right]\left(\partial_{i} \partial^{i}\right)$.

It is noted that the higher-order terms $\mathcal{O}(\beta)$ in the above equation are ignored. Adopting the effects of the generalized frequency $\bar{\omega}=E\left(1-\beta E^{2}\right)$ and the mass shell condition, the generalized expression of the energy is [56]

$\bar{E}=E\left[1-\beta\left(p^{2}+m^{2}\right)\right]$,

where the energy operator is defined as $E=i \hbar \partial_{t}$. Therefore, the original Klein-Gordon equation in the curved spacetime is given by

$\left[(i \hbar)^{2} D^{\mu} D_{\mu}+m^{2}\right] \Psi=0$,

where $D_{\mu}=\nabla_{\mu}+i e A_{\mu} / \hbar$ with the geometrically covariant derivative $\nabla_{\mu} ; m$ and $e$ denote the mass and charge of the particles, $A^{\mu}$ is the electromagnetic potential of spacetime. In order to get the generalized Klein-Gordon equation, Eq. (6) should be rewritten as

$$
\begin{aligned}
& -(i \hbar)^{2}\left(\partial_{t}+\frac{i}{\hbar} e A_{t}\right)\left(\partial^{t}+\frac{i}{\hbar} e A^{t}\right) \Psi \\
& =\left[(i \hbar)^{2}\left(\partial_{k}+\frac{i}{\hbar} e A_{k}\right)\left(\partial^{k}+\frac{i}{\hbar} e A^{k}\right)+m^{2}\right] \Psi,
\end{aligned}
$$

where $k=1,2,3 \ldots$ represent the spatial coordinates. In the above equation, the relation $\nabla_{\mu}=\partial_{\mu}$ has been used. The 
right hand of Eq. (7) is related to the energy. Inserting Eqs. (4) and (5) into the above equation, one can generalize the original Klein-Gordon equation to the following form:

$$
\begin{aligned}
- & (i \hbar)^{2}\left(\partial_{t}+\frac{i}{\hbar} e A_{t}\right)\left(\partial^{t}+\frac{i}{\hbar} e A^{t}\right) \Psi \\
= & {\left[(i \hbar)^{2}\left(\partial_{k}+\frac{i}{\hbar} e A_{k}\right)\left(\partial^{k}+\frac{i}{\hbar} e A^{k}\right)+m^{2}\right] } \\
& \times\left[1-\beta\left(p^{2}+m^{2}\right)\right]^{2} \Psi .
\end{aligned}
$$

The wave function of the generalized Klein-Gordon equation (Eq. (8)) can be expressed as $\Psi=\exp [i S(t, k) / \hbar]$, where $S(t, k)$ is the action of the scalar particle. Substituting the wave function into Eq. (8) and using the WKB approximation, the modified Hamilton-Jacobi equation for the scalar particle is got as

$$
\begin{gathered}
g^{00}\left(\partial_{0} S+e A_{0}\right)^{2}+\left[g^{k k}\left(\partial_{k} S+e A_{k}\right)^{2}+m^{2}\right] \\
\times\left\{1-2 \beta\left[g^{j j}\left(\partial_{j} S\right)^{2}+m^{2}\right]\right\}=0 .
\end{gathered}
$$

It is well known that the original Dirac equation can be expressed as $-i \gamma^{t} \nabla_{t} \Psi=\left(i \gamma^{k} \nabla_{k}+m / \hbar\right) \Psi$ with $\nabla_{k}=$ $\partial_{k}+\Omega_{k}+i e A_{k} / \hbar$, where the left hand is related to the energy. According to the method in $[42,43]$, putting the generalized expression of the energy, Eqs. (4) and (5), into the original Dirac equation, one finds the generalized Dirac equation in curved spacetime,

$-i \gamma^{t} \nabla_{t} \Psi=\left(i \gamma^{k} \nabla_{k}+m / \hbar\right) \Upsilon(\beta) \Psi$,

where $\Upsilon(\beta)=1-\beta\left(p^{2}+m^{2}\right)$. Since the $t$ - $t$ component of Eq. (10) is related to the energy, it did not get corrected by the GUP term $\Upsilon(\beta)$, thus Eq. (10) is different from the generalized Dirac equation $-i \gamma^{0} \partial_{0} \Psi=$ $\left(i \gamma^{i} \nabla_{i}+i \gamma^{t} \Omega_{t}+i e A_{t} / \hbar+m / \hbar\right) \Upsilon(\beta) \Psi$ in $[42,43]$. Then, multiplying $-i \gamma^{t} \nabla_{t}-\left[i \gamma^{n} \nabla_{n}-m / \hbar\right] \Upsilon(\beta)$ by Eq. (10), the generalized Dirac equation can be written as

$$
\begin{aligned}
& \left\{-\left(\gamma^{t} \nabla_{t}\right)^{2}-\gamma^{t} \nabla_{t} \gamma^{n} \nabla_{n} \Upsilon(\beta)-\gamma^{k} \nabla_{k} \gamma^{t} \nabla_{t}\right. \\
& \quad \times \Upsilon(\beta)+\left[i\left(\gamma^{n} \nabla_{n}-\gamma^{k} \nabla_{k}\right) \frac{m}{\hbar}-\gamma^{k} \nabla_{k} \gamma^{n} \nabla_{n}\right. \\
& \left.\left.-\left(\frac{m}{\hbar}\right)^{2}\right] \Upsilon(\beta)^{2}\right\} \Psi=0 .
\end{aligned}
$$

Assuming $k=n$, the above equation becomes

$$
\begin{aligned}
& \left\{-\frac{\left\{\gamma^{t}, \gamma^{t}\right\}}{2} \nabla_{t}^{2}-\left[\frac{\left\{\gamma^{k}, \gamma^{k}\right\}}{2} \nabla_{k}^{2}+\left(\frac{m}{\hbar}\right)^{2}\right] \Upsilon(\beta)^{2}\right\} \\
& \times \Psi=0 .
\end{aligned}
$$

In order to derive the modified Hamilton-Jacobi equation from Eq. (12), the wave function of the generalized Dirac equation takes the form
$\Psi=\xi(t, k) \exp [i S(t, k) / \hbar]$,

where $\xi(t, k)$ is a vector function of the spacetime. Denoting $t=0$, the gamma matrices' anti-commutation relations obey $\left\{\gamma^{0}, \gamma^{k}\right\}=0,\left\{\gamma^{k}, \gamma^{k}\right\}=2 g^{k k} I$, and $\left\{\gamma^{0}, \gamma^{0}\right\}=2 g^{00} I$. Substituting the gamma matrices' anti-commutation relations and Eq. (13) into Eq. (12), the resulting equation to leading order in $\beta$ is

$$
\begin{gathered}
\left\{g^{00}\left(\partial_{t} S+e A_{t}\right)^{2}+\left[g^{k k}\left(\partial_{k} S+e A_{k}\right)^{2}+m^{2}\right]\right. \\
\left.\quad \times\left\{1-2 \beta\left[g^{j j}\left(\partial_{j} S\right)^{2}+m^{2}\right]\right\}\right\} \xi(t, k)=0 .
\end{gathered}
$$

Equation (14) for the coefficient has a non-trivial solution if and only if the determinant vanishes, that is,

$$
\begin{aligned}
& \operatorname{Det}\left\{g^{00}\left(\partial_{t} S-e A_{t}\right)^{2}+\left[g^{k k}\left(\partial_{k} S+e A_{k}\right)^{2}+m^{2}\right]\right. \\
& \left.\quad \times\left\{1-2 \beta\left[g^{j j}\left(\partial_{j} S\right)^{2}+m^{2}\right]\right\}\right\}=0 .
\end{aligned}
$$

When keeping the leading-order term of $\beta$, the modified Hamilton-Jacobi equation for a fermion is directly obtained:

$$
\begin{aligned}
& g^{00}\left(\partial_{0} S+e A_{0}\right)^{2}+\left[g^{k k}\left(\partial_{k} S+e A_{k}\right)^{2}+m^{2}\right] \\
& \quad \times\left\{1-2 \beta\left[g^{j j}\left(\partial_{j} S\right)^{2}+m^{2}\right]\right\}=0 .
\end{aligned}
$$

Comparing Eq. (9) with Eq. (16), it is clear that the modified Hamilton-Jacobi equations for a scalar particle and fermions are similar. In $[37,46,57]$, the authors derived the HamiltonJacobi from the Rarita-Schwinger equation, the Maxwell equations, and the gravitational wave equation, and they indicated that the Hamilton-Jacobi equation can describe the behavior of particles with any spin in curved spacetime. As is well known, the Hamilton-Jacobi ansatz can greatly simplify the research of black hole radiation. Especially for the fermion tunneling case, we do not need to construct the tetrads and gamma matrices with the help of the HamiltonJacobi equation. Adopting the modified Hamilton-Jacobi equation, the tunneling radiation of ST black hole will be studied in the next section.

\section{Quantum tunneling from ST black hole}

To begin with, we need to make a few remarks about the ST black hole. In [58], the author added extra compact spatial dimensions to a static spherically symmetric spacetime, then he obtained the line element of the ST black hole

$\mathrm{d} s^{2}=-f(r) \mathrm{d} t^{2}+f(r)^{-1} \mathrm{~d} r^{2}+r^{2} \mathrm{~d} \Omega_{D-2}^{2}$,

where $f(r)=1-\left(r_{H} / r\right)^{D-3}, \mathrm{~d} \Omega_{D-2}^{2}$ is the metric on a unit $D-2$ dimensional sphere, covered by the original angular coordinates $\theta_{1}, \theta_{2}, \theta_{3}, \ldots, \theta_{D-2} . r_{H}$ is the event horizon of the ST black hole, which is characterized by the mass $M$, 


$$
\begin{aligned}
r_{H} & =\left[16 \pi G M /(D-2) \varpi_{D-2}\right]^{1 / D-3} \\
& =\frac{1}{\sqrt{\pi}}\left[8 M \Gamma\left(\frac{D-1}{2}\right) /(D-2) M_{P}^{D-2}\right]^{1 / D-3},
\end{aligned}
$$

where $G=1 / M_{P}^{D-2}$ is the $D-2$ dimensional Newton constant and the volume of the unit $D-2$ dimensional sphere is $\varpi_{D-2}=2 \pi^{\frac{D-1}{2}} / \Gamma\left(\frac{D-1}{2}\right)[48]$.

Next, we will calculate the quantum tunneling from the ST black hole. Inserting the inverse metric of ST black hole into the modified Hamilton-Jacobi equation, one has

$$
\begin{aligned}
& f^{-1}\left(\partial_{t} S\right)^{2}-\left[f(r)\left(\partial_{r} S\right)^{2}+\left(g^{\theta_{1} \theta_{1}}\right)\left(\partial_{\theta_{1}} S\right)^{2}\right. \\
& \quad+\left(g^{\theta_{2} \theta_{2}}\right)\left(\partial_{\theta_{2}} S\right)^{2}+\cdots+\left(g^{\theta_{D-2} \theta_{D-2}}\right)\left(\partial_{\theta_{D-2}} S\right)^{2} \\
& \left.\quad+m^{2}\right]\left\{1-2 \beta\left[f(r)\left(\partial_{r} S\right)^{2}+\left(g^{\theta_{1} \theta_{1}}\right)\left(\partial_{\theta_{1}} S\right)^{2}\right.\right. \\
& \quad+\left(g^{\theta_{2} \theta_{2}}\right)\left(\partial_{\theta_{2}} S\right)^{2}+\cdots+\left(g^{\theta_{D-2} \theta_{D-2}}\right)\left(\partial_{\theta_{D-2}} S\right)^{2} \\
& \left.\left.\quad+m^{2}\right]\right\}=0 .
\end{aligned}
$$

Since the spacetime of ST black hole is static, the action $S$ is supposed to take the form $S=-\omega t+W(r)+$ $\Theta\left(\theta_{1}, \theta_{2}, \ldots, \theta_{D-2}\right)$, where $\omega$ is the energy of the emitted particles. Equation (19) can be written as

$$
\begin{aligned}
2 \beta f^{2} & (r)\left(\partial_{r} W\right)^{4}+\omega^{2} f^{-1}(r)+2 m^{4} \beta+f(r) \\
& \times\left(4 m^{2} \beta-1\right)\left(\partial_{r} W\right)^{2}+\left[4 m^{2} \beta+4 \beta f(r)\right. \\
& \left.\times\left(\partial_{r} W\right)^{2}-1\right]\left[\left(g^{\theta_{1} \theta_{1}}\right)\left(\partial_{\theta_{1}} \Theta\right)^{2}+\left(g^{\theta_{2} \theta_{2}}\right)\left(\partial_{\theta_{2}} \Theta\right)^{2}\right. \\
& \left.+\left(g^{\theta_{D-2} \theta_{D-2}}\right)\left(\partial_{D-2} \Theta\right)^{2}\right]-m^{2}=-\lambda,
\end{aligned}
$$

$$
\begin{aligned}
& 2 \beta\left[\left(g^{\theta_{1} \theta_{1}}\right)\left(\partial_{\theta_{1}} \Theta\right)^{2}+\left(g^{\theta_{2} \theta_{2}}\right)\left(\partial_{\theta_{2}} \Theta\right)^{2}\right. \\
& \left.+\cdots+\left(g^{\theta_{D-2} \theta_{D-2}}\right)\left(\partial_{\theta_{D-2}} \Theta\right)^{2}\right]^{2}=\lambda,
\end{aligned}
$$

where $\lambda$ is a constant. First, focusing on Eq. (21), in [46], the author showed that the magnitude of the particles' angular momentum can be expressed in terms of $\partial_{\theta_{1}} \Theta, \partial_{\theta_{2}} \Theta$, $\ldots \partial_{\theta_{D-2}} \Theta$, that is,

$$
\begin{aligned}
& \left(g^{\theta_{1} \theta_{1}}\right)\left(\partial_{\theta_{1}} \Theta\right)^{2}+\left(g^{\theta_{2} \theta_{2}}\right)\left(\partial_{\theta_{2}} \Theta\right)^{2} \\
& +\cdots+\left(g^{\theta_{D-2} \theta_{D-2}}\right)\left(\partial_{D-2} \Theta\right)^{2}=\mathcal{L}^{2} .
\end{aligned}
$$

According to Eq. (22), one can write Eq. (21) as

$2\left(\mathcal{L}^{2}\right)^{2}=\lambda / \beta$

In the above equation is indicated that the constant $\lambda$ is related to the angular momentum of the emitted particle. With the help of Eqs. (22) and (23), Eq. (20) becomes

$$
P_{4}\left(\partial_{r} W\right)^{4}+P_{2}\left(\partial_{r} W\right)^{2}+P_{0}=0,
$$

where $P_{4}=2 \beta f(r)^{2}, P_{2}=f(r)\left(4 m^{2} \beta+\sqrt{8 \beta \lambda}-1\right)$ and $P_{0}=\omega^{2} f^{-1}(r)+m^{2}\left[2 \beta m^{2}+(\sqrt{8 \beta \lambda}-1)\right]-\sqrt{\lambda / 2 \beta}+\lambda$.
Neglecting the higher orders $\beta$ and solving the above equation, one finds

$$
\begin{aligned}
W_{ \pm}= & \pm \frac{1}{f(r)} \sqrt{f(r)\left(m^{2}-\lambda+\sqrt{\lambda / 2 \beta}\right)+\omega^{2}} \\
& \times\left\{1+\beta\left[m^{2}+f^{-1}(r) \omega^{2}\right]+\sqrt{\beta \lambda / 2}\right\} \mathrm{d} r
\end{aligned}
$$

where the $+/-$ denote the outgoing/incoming solutions of the emitted particles. In order to solve the above equation, one needs to find the residue of Eq. (25) on the event horizon. By expanding a Laurent series on the event horizon and keeping the first-order term of $\beta$, the result of Eq. (25) takes the form

$$
\begin{aligned}
W\left(r_{H}\right)_{ \pm}= & \pm \frac{i \pi r_{H} \omega}{D-3}\left\{1+\sqrt{\frac{\beta \lambda}{8}}+\beta\right. \\
& \left.\times\left[\frac{m^{2}+\lambda}{2}+\frac{(D-2) \omega^{2}}{(D-3)}\right]\right\}+\Delta \text { (realpart) }
\end{aligned}
$$

Because the real part of Eq. (26) is irrelevant to the tunneling rate, we only keep the imaginary part. For obtaining the tunneling rate from Eq. (26), one needs to solve the factor-2 problem $[59,60]$. One of the best ways to solve this problem is to adopt a temporal contribution expression. According to [61-65], the spatial part of the tunneling rate of the emitted particle is

$$
\begin{aligned}
\Gamma & \propto \exp \left(-\operatorname{Im} \oint p_{r} \mathrm{~d} r\right)=\exp \left[\operatorname{Im}\left(\int p_{r}^{\text {out }} \mathrm{d} r-\int p_{r}^{\text {in }} \mathrm{d} r\right)\right] \\
& =\exp \left\{-\frac{2 \pi r_{H} \omega}{D-3}\left\{1+\sqrt{\frac{\beta \lambda}{8}}+\beta\left[\frac{m^{2}+\lambda}{2}+\frac{(D-2) \omega^{2}}{(D-3)}\right]\right\}\right\},
\end{aligned}
$$

where $p_{r}=\partial_{r} W$. However, as pointed out in [62], the temporal contribution to the tunneling amplitude was lost in the above discussion. For incorporating the temporal contribution into our calculation, we need to use Kruskal coordinates $(T, R)$. The exterior region is given by

$T=\exp \left(\kappa r_{*}\right) \sinh (\kappa t), \quad R=\exp \left(\kappa r_{*}\right) \cosh (\kappa t)$,

where $r_{*}=r+\frac{1}{2 \kappa} \ln \frac{r-r_{H}}{r_{H}}$ is the tortoise coordinate and $\kappa$ is the surface gravity of the ST black hole. In order to connect the interior region and the exterior region across the horizon, one can rotate the time as $t \rightarrow t-i \pi / 2 \kappa$. By this operation, one obtains an additional imaginary contribution $\operatorname{Im}\left(\omega \Delta t^{\text {out,in }}\right)=\omega \pi / 2 \kappa$. Therefore, the total temporal contribution becomes $\operatorname{Im} \omega \Delta t=\omega \pi / \kappa$. Putting this contribution into Eq. (27), the GUP corrected tunneling rate of the emitted particle across the horizon is derived to be 


$$
\begin{aligned}
\Gamma & \propto \exp \left[-\operatorname{Im}\left(\omega t+\operatorname{Im} \oint p_{r} d r\right)\right] \\
& =\exp \left\{-\frac{4 \pi r_{H} \omega}{D-3}\left\{1+\sqrt{\frac{\beta \lambda}{8}}+\beta\left[\frac{m^{2}+\lambda}{2}+\frac{(D-2) \omega^{2}}{(D-3)}\right]\right\}\right\} .
\end{aligned}
$$

Employing the Boltzmann factor, the GUP corrected Hawking temperature is

$$
T_{H}=T_{0}\left\{1+\sqrt{\frac{\beta \lambda}{8}}+\beta\left[\frac{1}{2}\left(m^{2}+\lambda\right)+\frac{(D-2) \omega^{2}}{(D-3)}\right]\right\}^{-1}
$$

where $T_{0}=(D-3) / 4 \pi r_{H}$ is the original Hawking temperature of the ST black hole. Now, we turn to the calculation of the entropy of the ST black hole. Based on the first law of black hole thermodynamics, the entropy can be expressed as

$$
\begin{aligned}
S= & \int T_{H}^{-1} \mathrm{~d} M=\int \frac{4 \pi r_{H}}{D-3}\left[\frac{(D-2) \varpi}{16 \pi G M}\right]^{\frac{1}{D-3}} \\
& \times\left\{1+\sqrt{\frac{\beta \lambda}{8}}+\beta\left[\frac{1}{2}\left(m^{2}+\lambda\right)+\frac{(D-2) \omega^{2}}{(D-3)}\right]\right\} \mathrm{d} M .
\end{aligned}
$$

The above equation cannot be evaluated exactly for general $D$. According to the original Hawing radiation theory, all particles near the event horizon seem effectively massless. Therefore, we do not consider the mass of the emitted particles in the following discussion.

\section{Remnants of ST black hole}

A lot of work showed that the GUP can lead to a black hole remnant [24-28,40-47]. Therefore, it is interesting to investigate the remnant of the ST black hole. According to the saturated form of the uncertainty principle, one gets a lower bound on the energy of the emitted particle in Hawking radiation, which can be expressed as $[23,66]$

$\omega \geq \hbar / \Delta x$

Near the event horizon of the ST black hole, it is possible to take the value of the uncertainty in position as the radius of the black hole $[23,66]$,

$\Delta x \approx r_{B H}=r_{H}$.
Putting Eqs. (32) and (33) into Eq. (30), one has

$$
\begin{aligned}
T_{H} & =T_{0}\left\{1+\frac{3}{2} \sqrt{\frac{\beta \lambda}{2}}+\beta\left[\frac{(D-2) \omega^{2}}{(D-3)}-\frac{\lambda}{2}\right]\right\}^{-1} \\
& \simeq T_{0}\left\{\frac{2\left[4(D-2) \hbar^{2} \beta+(D-3) r_{H}^{2}(\sqrt{2 \beta \lambda}+2 \beta \lambda-4)\right]}{r_{H}^{2}(D-3)(\beta \lambda-8)}\right\} .
\end{aligned}
$$

It is clear that $T_{H}$ sensitively depends on the event horizon of the ST black hole, the spacetime dimension $D$, the angular momentum of the emitted particles, and the quantum gravity effect $\beta$. An important relation should be mentioned, when $r_{H}<\sqrt{\frac{4(D-2) \beta \hbar^{2}}{(D-3)(4-2 \beta \lambda-\sqrt{2 \beta \lambda})}}$, the Hawking temperature goes to negative values, and it violates the laws of black hole thermodynamics and has no physical meaning. Therefore, this relation indicates the existence of a minimum radius, where the Hawking temperature equals zero, that is,

$$
\begin{aligned}
r_{\min } & =\sqrt{\frac{4(D-2) \beta \hbar^{2}}{(D-3)(4-2 \beta \lambda-\sqrt{2 \beta \lambda})}} \\
& =\ell_{p} \sqrt{\frac{4 \hbar^{2}(D-2) \beta_{0}}{(D-3)\left(4 \hbar^{2}-2 \lambda \beta_{0} \ell_{p}^{2}-\ell_{p} \hbar \sqrt{2 \lambda \beta_{0}}\right)}} .
\end{aligned}
$$

In addition, we can also express Eq. (34) in terms of the mass of the ST black hole to obtain the temperature-mass relation,

$$
\begin{aligned}
T_{H} \simeq & \frac{D-3}{4 \pi}\left[\frac{(D-2) \varpi_{D-2}}{16 \pi G M}\right]^{\frac{1}{D-3}} \\
& \times \frac{2\left\{4 \hbar^{2} \beta(D-2)-(D-3)\left[\frac{(D-2) \varpi_{D-2}}{16 \pi G M}\right]^{\frac{2}{D-3}}(4-\sqrt{2 \beta \lambda}-2 \beta \lambda)\right\}}{(D-3)\left[\frac{(D-2) \varpi_{D-2}}{16 \pi G M}\right]^{\frac{2}{D-3}}(\beta \lambda-8)} .
\end{aligned}
$$

From Eq. (36), we find that the GUP corrected temperature has physical meaning as far as the mass of the ST black hole satisfies the inequality $M \geq \frac{(D-2) \varpi_{D-2}}{16 \pi G}$ $\left[\frac{4(D-2) \hbar^{2} \beta}{(D-3)(4-2 \beta \lambda-\sqrt{2 \beta \lambda})}\right]^{\frac{D-3}{2}}$, which implies that the mass of ST black hole has a minimum value,

$$
\begin{aligned}
M_{\min } & =\frac{(D-2) \varpi_{D-2}}{16 \pi G}\left[\frac{4(D-2) \hbar^{2} \beta}{(D-3)(4-2 \beta \lambda-\sqrt{2 \beta \lambda})}\right]^{\frac{D-3}{2}} \\
& =\frac{(D-2) M_{p}}{8 \Gamma\left(\frac{D-1}{2}\right)}\left[\frac{4 \pi \beta_{0} \hbar^{2}(D-2)}{c^{2}(D-3)\left(4-\frac{2 \lambda \beta_{0}}{M_{p}^{2} c^{2}}-\sqrt{\frac{2 \lambda \beta_{0}}{M_{p}^{2} c^{2}}}\right)}\right]^{\frac{D-3}{2}} .
\end{aligned}
$$

Obviously, the minimum mass is related to the Planck mass. According to Eqs. (36) and (37), the behaviors of the GUP 

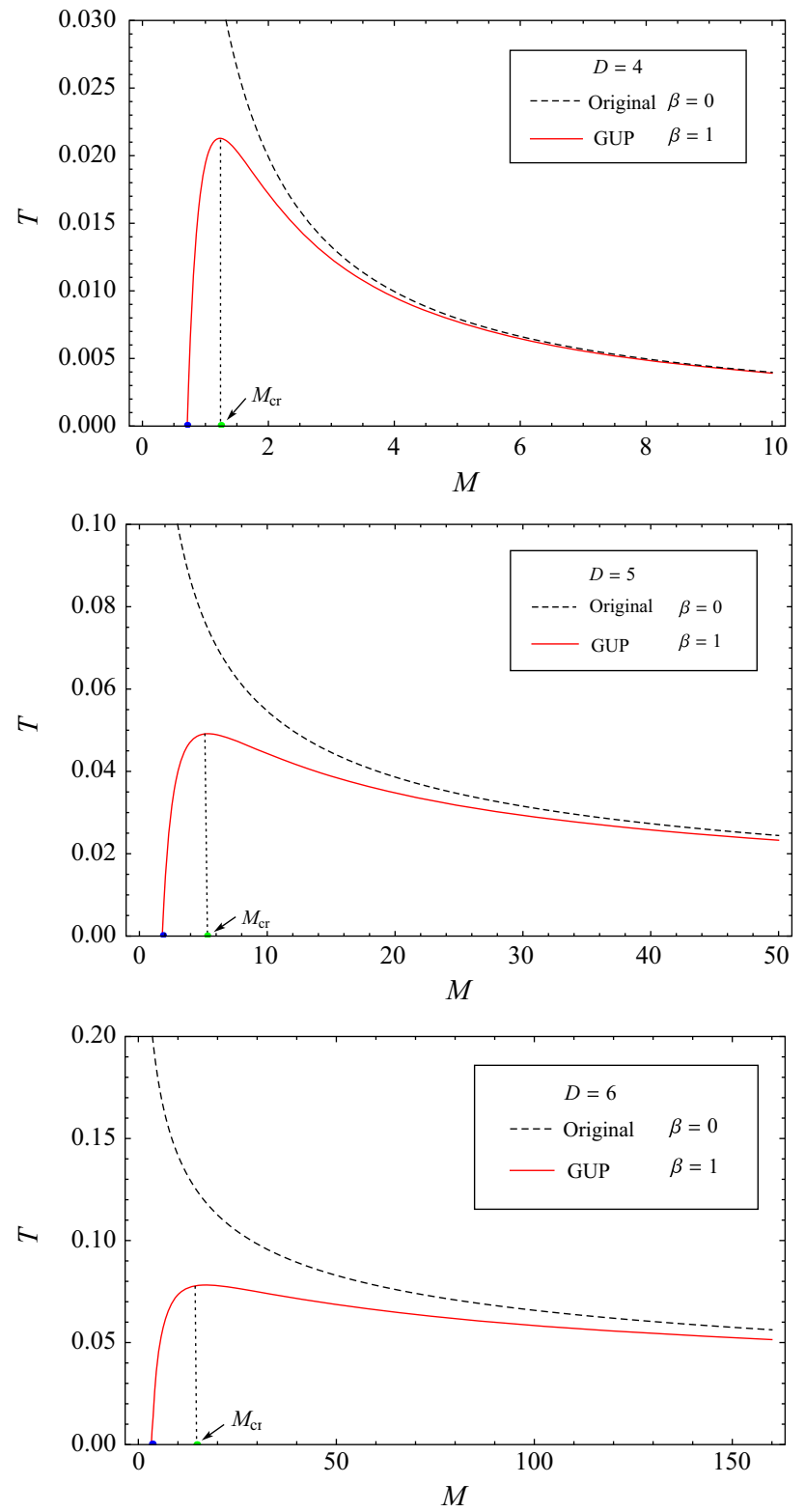

Fig. 1 The original and GUP corrected Hawking temperature of ST black hole for different values of mass. We set $M_{P}=c=\hbar=1$, $\lambda=0.001$, and $D=4,5,6$

corrected Hawking temperature and the original Hawking temperature of the ST black hole are plotted in Fig. 1.

In Fig. 1, the dashed black lines and solid red lines in the diagrams illustrate the original Hawking temperature and the GUP corrected temperature of the ST black hole. It is easy to see that the GUP corrected temperature is lower than the original Hawking temperature. Besides, different values of $D$ give a similar behavior of the Hawking temperature. For a large mass of the black hole, the GUP corrected temperature tends to the original value of the Hawking temperature because the effect of quantum gravity is negligible at that scale. However, as the mass of the black hole decreases, the
GUP corrected temperature reaches the maximum value (at the critical mass $M_{c r}$, which is marked by a green dot), and then decreases to zero when the mass approaches the minimum value of the mass $\left(M_{\min } \sim M_{p}\right.$, which is marked by a blue dot). The GUP corrected temperature is unphysical below $M_{\min }$, it signals the existence of a black hole remnant $M_{\text {res }}=M_{\text {min }}$. The black hole remnant can be further confirmed from the heat capacity.

Since the thermodynamic stability of black hole is determined by the heat capacity $\mathcal{C}$, further inspection of the existence of the black hole remnant can be made by investigating the heat capacity of the ST black hole. The GUP corrected heat capacity is given by

$\mathcal{C}=T_{H}\left(\frac{\partial S}{\partial T_{H}}\right)=T_{H}\left(\frac{\partial S}{\partial M}\right)\left(\frac{\partial T_{H}}{\partial M}\right)^{-1}=\frac{\mathcal{A}}{\mathcal{B}}$.

According to Eqs. (32) and (33), the entropy can be rewritten as

$$
\begin{aligned}
S= & \int \frac{4 \pi r_{H}}{D-3}\left[\frac{(D-2) \varpi}{16 \pi G M}\right]^{\frac{1}{D-3}}\left\{1+\sqrt{\frac{\beta \lambda}{8}}\right. \\
& \left.+\beta\left\{\frac{\lambda}{2}+\frac{(D-2) \omega^{2} \hbar^{2}}{(D-3)}\left[\frac{(D-2) \varpi}{16 \pi G M}\right]^{\frac{2}{D-3}}\right\}\right\} \mathrm{d} M
\end{aligned}
$$

and $\mathcal{A}$ and $\mathcal{B}$ in Eq. (38) are defined by

$$
\begin{aligned}
\mathcal{A}= & 2^{2+\frac{4}{D-3}}\left[\frac{G}{(D-2) \varpi}\right]^{\frac{1}{D-3}}(M \pi)^{1+\frac{1}{D-3}} \\
& \times\left(4 \hbar^{2} \beta \frac{(D-2)}{(D-3)}-\left[\frac{16 \pi G M}{(D-2) \varpi}\right]^{\frac{2}{D-3}}(4-\sqrt{2 \beta \lambda}-2 \beta \lambda)\right) \\
& \times\left[\left(1+\frac{\sqrt{2 \beta \lambda}}{4}+\frac{\beta \lambda}{2}\right)+\frac{\hbar^{2} \beta(D-2)^{1+\frac{2}{D-3}}}{D-3}\left(\frac{16 \pi G M}{\varpi}\right)^{\frac{2}{D-3}}\right],
\end{aligned}
$$

$$
\begin{aligned}
\mathcal{B}= & -\left\{\frac{12 \hbar^{2} \beta(D-2)}{D-3}+\left(2^{\frac{D+3}{D-3}}-3 \times 2^{\frac{6}{D-3}}\right)\right. \\
& \left.\times\left[\frac{2 G M \pi}{(D-2) \varpi}\right]^{\frac{2}{D-3}}(4-\sqrt{2 \beta \lambda}-2 \beta \lambda)\right\} .
\end{aligned}
$$

Assuming $\beta=0$, one obtains the original specific heat of the ST black hole from Eq. (38). We find that the specific heat goes to zero at $M=\frac{(D-2) \omega_{D-2}}{16 \pi G}\left[\frac{4(D-2) \hbar^{2} \beta}{(D-3)(4-2 \beta \lambda-\sqrt{2 \beta \lambda})}\right]^{\frac{D-3}{2}}$, which is equal to $M_{\min }$ by Eq. (37). The behaviors of the heat capacity of ST black hole for $D=4,5$, and 6 are shown in Fig. 2.

In Fig. 2, one can see the specific heat versus the mass of the ST black hole. Notably, the different values of $D$ give a similar behavior of specific heat. The black dashed lines correspond to the original specific heat, there are negative 

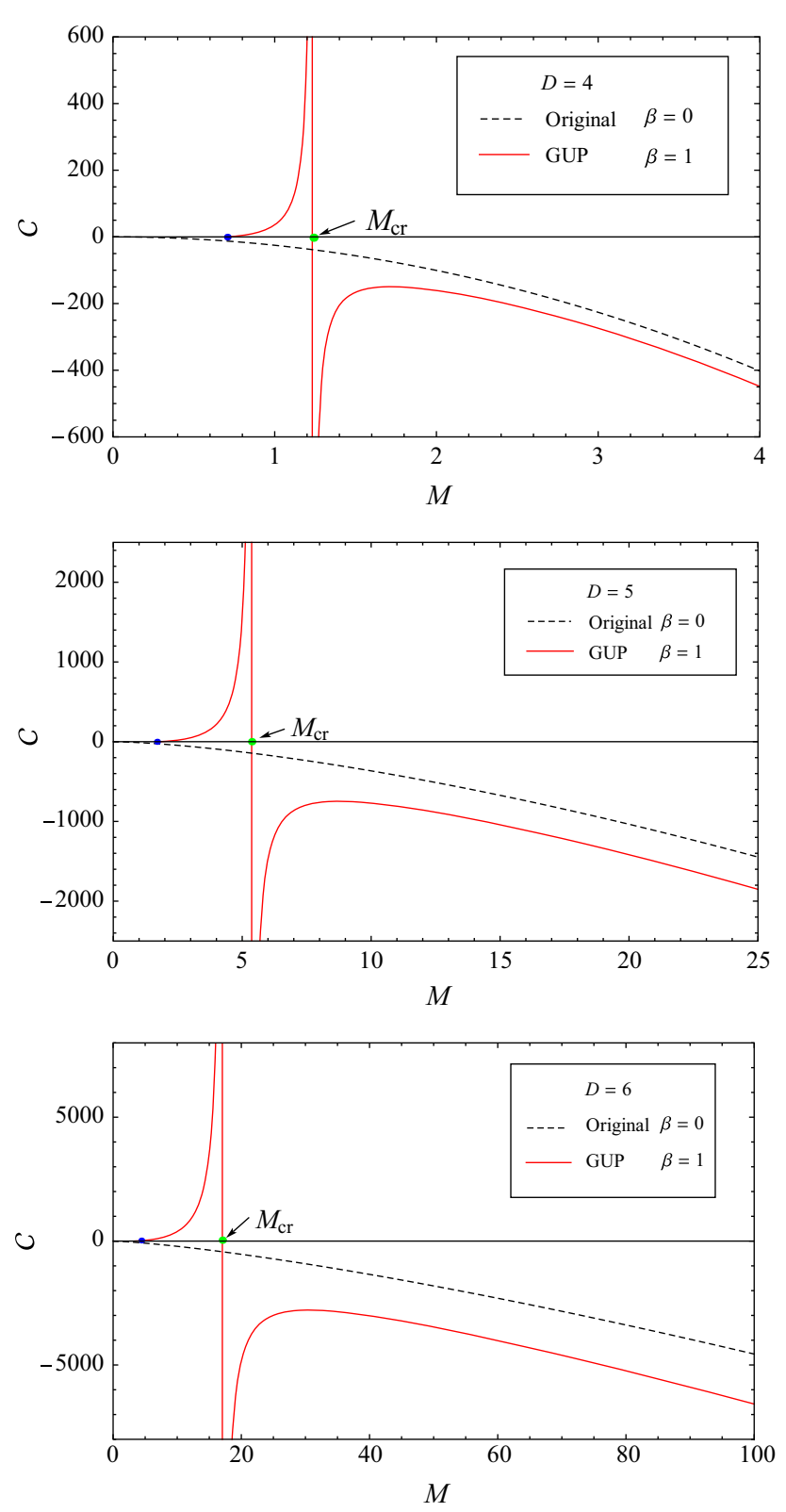

Fig. 2 The original and GUP corrected specific heat of the ST black hole for different values of the mass. We assumed $M_{P}=c=\hbar=1$, $\lambda=0.001$, and $D=4,5,6$

values going to zero when $M \rightarrow 0$. The GUP corrected specific heat is represented by red solid lines. It is clear that the GUP corrected specific heat diverges at the green dot, where the GUP corrected temperature reaches its maximum value $M_{c r}$. When the mass of the black hole is large enough, the behavior of the GUP corrected specific heat is similar to the original case. By decreasing the mass of the ST black hole, the GUP corrected specific heat becomes smaller and departs from the original ST black hole behavior. However, at $M=M_{c r}$, the GUP corrected specific heat has a vertical asymptote at a certain location; it implies a thermodynamic phase transition occurred from $\mathcal{C}<0$ (unstable phase) to $\mathcal{C}>$
Table 1 The latest experimental limits on $M_{p}$, the minimal energy for black hole formation $E_{\mathrm{min}}^{\mathrm{GUP}}$ and $E_{\mathrm{min}}^{\mathrm{GR}}$ in different dimensions $D$

\begin{tabular}{llll}
\hline$D$ & $M_{p}(\mathrm{TeV})$ & $E_{\mathrm{min}}^{\mathrm{GUP}}(\mathrm{TeV})$ & $E_{\mathrm{min}}^{\mathrm{GR}}(\mathrm{TeV})[73]$ \\
\hline 6 & 4.54 & 14.6 & 9.5 \\
7 & 3.51 & 17.0 & 10.8 \\
8 & 2.98 & 18.7 & 11.8 \\
9 & 2.71 & 19.7 & 12.3 \\
10 & 2.51 & 19.2 & 11.9 \\
\hline
\end{tabular}

0 (stable phase), and this phase transition is also found in the GUP black holes [67] and the framework of gravity's rainbow [68-70]. Finally, the GUP corrected specific heat decreases to zero as the mass decreases to $M_{\min }$ (blue dot). The $\mathcal{C}=0$ means that the black hole cannot exchange its energy with environment, hence the GUP stops the evolution of black holes at this point and leads to the black hole remnant, that is, $M_{\min }=M_{\text {res }}$.

\section{The minimal energy for $\mathrm{BH}$ formation in colliders}

The production of black holes at colliders such as LHC is one of the most exciting predictions of physics. Due to Eq. (37), one can calculate whether the black holes could be formed at the LHC. The minimum energy needed to form a black hole in a collider is given by

$$
\begin{aligned}
& E_{\mathrm{min}}^{\mathrm{GUP}} \\
& \quad=\frac{(D-2) M_{p}}{8 \Gamma\left(\frac{D-1}{2}\right)}\left[\frac{4 \pi \beta_{0} \hbar^{2}(D-2)}{c^{2}(D-3)\left(4-\frac{2 \lambda \beta_{0}}{M_{p}^{2} c^{2}}-\sqrt{\frac{2 \lambda \beta_{0}}{M_{p}^{2} c^{2}}}\right)}\right]^{\frac{D-3}{2}} .
\end{aligned}
$$

In order to investigate the minimal energy for black hole formation, we use the latest observed limits on the ADD model [71] parameter $M_{p}$ with a next-to-leading-order (NLO) $K$ factor [72,73]. When setting $\beta_{0}=c=\hbar=1$ and $\lambda=0.001$, the minimum energy to form a black hole, $E_{\mathrm{min}}^{\mathrm{GUP}}$, is shown in Table 1.

We also compare our results with the results obtained in the theory of Gravity's Rainbow $(\mathrm{GR}) E_{\min }^{\mathrm{GR}}=\frac{(D-2)}{8 \Gamma\left(\frac{D-1}{2}\right)} \pi^{\frac{D-3}{2}}$ $\eta^{\frac{D-3}{n}} M_{p}$, where $\eta(=1)$ and $n(=2)$ represent the rainbow parameter and an integer; $M_{p}$ is the Planck mass [73]. It is shown that our results are higher than $E_{\text {min }}^{\mathrm{GR}}$. This difference is caused by different modified gravity theories. Quite recently, the protons collided in the LHC have reached the new energy regime of $13 \mathrm{TeV}$ [74], but it is still smaller than the $E_{\mathrm{min}}^{\mathrm{GUP}}$ in $D=6$, which implies the black hole cannot be 
Table 2 The lower bounds on $\beta_{0}$ for different $D$, we set $c=\hbar=1$ and $\lambda=0.001$

\begin{tabular}{llllll}
\hline$D$ & 6 & 7 & 8 & 9 & 10 \\
\hline$\beta_{0}$ & 0.9216 & 0.8740 & 0.8642 & 0.8707 & 0.8944 \\
\hline
\end{tabular}

produced in the LHC. This may explain the absence of black holes in the current LHC.

Moreover, we only fix $\beta_{0}=1$ in Table 1 . However, from the expression of $E_{\min }$, we find it is closely related to the dimensionless constant $\beta_{0}$, which indicates that the different value of $\beta_{0}$ may lead to different values of the minimum energy for black hole formation. The lower bound of $\beta_{0}$ can be studied by the following formula:

$$
\begin{gathered}
\beta_{0}>4 \chi-\frac{2 \chi^{3} \lambda^{2}}{\left(c^{2} M_{p}^{2}+2 \lambda \chi\right)^{2}}-\frac{7 \lambda \chi^{2}}{c^{2} M_{p}^{2}+2 \lambda \chi} \\
-\frac{c^{2} M_{p}^{2} \chi \sqrt{\chi \lambda\left(8 c^{2} M_{p}^{2}+17 \chi \lambda\right)}}{\left(c^{2} M_{p}^{2}+2 \chi \lambda\right)^{2}}
\end{gathered}
$$

where $\chi=\frac{c^{2}(D-3)}{4 \pi \hbar^{2}(D-2)}\left[\frac{13 \mathrm{TeV} \times 8 \Gamma\left(\frac{D-1}{2}\right)}{(D-2) M_{p}}\right]^{\frac{2}{D-3}}$. The bounds on $\beta_{0}$ for $D=6,7,8,9,10$ are given in Table 2. Combining our results with earlier versions of GUP and some phenomenological implications in [10,75-77], it indicates that $\beta_{0} \sim 1$.

\section{Conclusions}

In this work, we have investigated the GUP effect on the thermodynamics of ST black hole. First of all, we derived the modified Hamilton-Jacobi equation by employing the GUP with a quadratic term in momentum. With the help of the modified Hamilton-Jacobi equation, the quantum tunneling from an ST black hole has been studied. Finally, we obtained the GUP corrected Hawking temperature, entropy, and heat capacity. For the original Hawing radiation, the Hawking temperature of the ST black hole is related to its mass. However, our results showed that if the effect of quantum gravity is considered, the behavior of the tunneling particle on the event is different from the original case, and the GUP corrected thermodynamic quantities are not only sensitively dependent on the mass $M$ and the spacetime dimension $D$ of ST black hole, but also on the angular momentum parameter $\lambda$ and the quantum gravity term $\beta$. Besides, we found that the GUP corrected Hawking temperature is smaller than the original case; it goes to zero when the mass of ST black hole reaches the minimal value $M_{\min }$, which is of the order of the
Planck scale, and it predicts the existence of a black hole remnant. For confirming the black hole remnant, the GUP corrected heat capacity has also been analyzed. It was shown that the GUP corrected heat capacity has a phase transition at $M_{\mathrm{cr}}$, where the GUP corrected temperature reaches its maximum value; then the GUP corrected heat vanishes when the mass approaches $M_{\min }$ in the final stages of black hole evaporation. At this point, the ST black hole does not exchange energy with the environment, hence the remnant of ST black hole is produced. The reason for this remnant is related to the fact that the quantum gravity effect is running as the size of the black hole approaches the Planck scale. The existence of a black hole remnant implies that black holes would not evaporate, its information and singularity are enclosed in the event horizon. Finally, we discussed the minimum energy to form a black hole in the LHC. The results showed that the minimum energy to form a black hole in our work is larger than the current energy scales of LHC, and this may explain why one cannot observe a black hole in the LHC. Our results are supported by the results obtained in the framework of gravity's rainbow [68-70]. Therefore, we think that the GUP effect can effectively prevent a black hole from evaporating completely, and this may solve the information loss and naked singularity problems of black holes $[78,79]$.

Acknowledgments This work is supported by the Natural Science Foundation of China (Grant No. 11573022).

Open Access This article is distributed under the terms of the Creative Commons Attribution 4.0 International License (http://creativecomm ons.org/licenses/by/4.0/), which permits unrestricted use, distribution, and reproduction in any medium, provided you give appropriate credit to the original author(s) and the source, provide a link to the Creative Commons license, and indicate if changes were made.

Funded by $\mathrm{SCOAP}^{3}$.

\section{References}

1. K. Konishi, G. Paffuti, P. Provero, Phys. Lett. B 234, 276 (1990). doi:10.1016/0370-2693(90)91927-4

2. M. Maggiore, Phys. Lett. B 319, 83 (1993). arXiv:hep-th/9309034

3. L.J. Garay, Int. J. Mod. Phys. A 10, 145 (1995). arXiv:gr-qc/9403008

4. G. Amelino-Camelia, Int. J. Mod. Phys. D 11, 35 (2002). arXiv:gr-qc/0012051

5. F. Scardigli, Phys. Lett. B 452, 39 (1999). arXiv:hep-th/9904025

6. A. Kempf, J. Phys. A Math. Gen. 30, 2093 (1997). arXiv:hep-th/9604045

7. F. Brau, J. Phys. A: Math. Gen. 32, 7691 (1999). arXiv:quant-ph/9905033

8. J. Magueijo, L. Smolin, Phys. Rev. D 67, 044017 (2003). arXiv:gr-qc/0207085

9. J. Magueijo, L. Smolin, Class. Quantum Gravity 21, 1725 (2004). arXiv:gr-qc/0305055

10. S. Das, E.C. Vagenas, Phys. Rev. Lett. 101, 221301 (2008). arXiv: 0810.5333

11. M. Sprenger, P. Nicolini, M. Bleicher, Eur. J. Phys. 33, 853 (2012). arXiv: 1202.1500 
12. S. Hossenfelder, Living Rev. Rel. 16, 2 (2013). arXiv:1203.6191

13. G. Amelino-Camelia, Living Rev. Rel. 16, 5 (2013). arXiv:0806.0339

14. A. Kempf, G. Mangano, R.B. Mann, Phys. Rev. D 52, 1108 (1995). arXiv:hep-th/9412167

15. S. Das, R.B. Mann, Phys. Lett. B 704, 596 (2011). arXiv: 1109.3258

16. M. Sprenger, M. Bleicher, P. Nicolini, Class. Quantum Gravity 28, 235019 (2011). arXiv:1011.5225

17. A.F. Ali, S. Das, E.C. Vagenas, Phys. Rev. D 84, 044013 (2011). arXiv: 1107.3164

18. T. Zhu, J.R. Ren, M.F. Li, Phys. Lett. B 674, 204 (2009). arXiv:0811.0212

19. W. Chemissany, S. Das, A.F. Ali, E.C. Vagenas, JCAP 1112, 017 (2011). arXiv:1111.7288

20. A.F. Ali, Class. Quantum Gravity 28, 065013 (2011). arXiv: 1101.4181

21. A.F. Ali, A. Tawfik, Adv. High Energy Phys. 2013, Article ID 126528 (2013). arXiv: 1301.3508

22. A. Tawfik, A. Diab, Int. J. Mod. Phys. D 23, 1430025 (2014). arXiv: 1410.0206

23. R.J. Adler, P. Chen, D.I. Santiago, Gen. Relativ. Gravit. 33, 2101 (2001). arXiv:gr-qc/0106080

24. A.J.M. Medved, E.C. Vagenas, Phys. Rev. D 70, 124021 (2004). arXiv:hep-th/0411022

25. Y.S. Myung, Y.W. Kim, Y.J. Park, Phys. Lett. B 645, 393 (2007). arXiv:gr-qc/0609031

26. M.I. Park, Phys. Lett. B 659, 698 (2008). arXiv:0709.2307

27. S. Gangopadhyay, A. Dutta, A. Saha, Gen. Relativ. Gravit. 46, 1661 (2014). arXiv:1307.7045

28. S. Gangopadhyay, A. Dutta, M. Faizal, EPL 112, 20006 (2015). arXiv: 1501.01482

29. M.K. Parikh, F. Wilczek, Phys. Rev. Lett. 85, 5042 (2000). arXiv:hep-th/9907001

30. J. Zhang, Z. Zhao, JHEP 10, 055 (2005). doi:10.1088/1126-6708/ 2005/10/055

31. K. Srinivasan, T. Padmanabhan, Phys. Rev. D 60, 24007 (1999). arXiv:gr-qc/9812028

32. S. Shankaranarayanan, Phys. Rev. D 67, 084026 (2003). arXiv:gr-qc/0301090

33. J.Y. Zhang, Z. Zhao, Phys. Lett. B 638, 110 (2006). arXiv:gr-qc/0512153

34. R. Kerner, R.B. Mann, Class. Quantum Gravity 25, 095014 (2008). arXiv:0710.0612

35. Q.Q. Jiang, S.Q. Wu, X. Cai, Phys. Rev. D 73, 064003 (2006). arXiv:hep-th/0512351

36. D.Y. Chen, Q.Q. Jiang, X.T. Zu, Phys. Lett. B 665, 106 (2008). arXiv:0804.0131

37. A. Yale, R.B. Mann, Phys. Lett. B 673, 168 (2009). arXiv:0808.2820

38. H.L. Li, R. Lin, Gen. Relativ. Gravit. 43, 2115 (2011). doi:10.1007/ s10714-011-1169-7

39. Z.W. Feng, Y. Chen, X.T. Zu, Astrophys. Space Sci. 48, 359 (2015). doi:10.1007/s10509-015-2498-X

40. K. Nozari, S.H. Mehdipour, EPL 84, 20008 (2008). arXiv:0804.4221

41. B. Majumder, Gen. Relativ. Gravit. 45, 2403 (2013). arXiv: 1212.6591

42. D.Y. Chen, H.W. Wu, H.T. Yang, Adv. High Energy Phys. 2013, Article ID 432412 (2013). arXiv: 1305.7104

43. D.Y. Chen, Q.Q. Jiang, P. Wang, H.T. Yang, JHEP 11, 176 (2013). arXiv: 1312.3781

44. D.Y. Chen, H.W. Wu, H.T. Yang, JCAP 03, 036 (2014). arXiv: 1307.0172

45. P. Bargueño, E.C. Vagenas, Phys. Lett. B 742, 15 (2015). arXiv:1501.03256
46. B.R. Mu, P. Wang, H.T. Yang, Adv. High Energy Phys. 2015, Article ID 898916 (2015). arXiv: 1501.06025

47. M.A. Anacleto, F.A. Brito, E. Passos, Phys. Lett. B 749, 181 (2015). arXiv:1504.06295

48. S. Dimopoulos, G.L. Landsberg, Phys. Rev. Lett. 87, 161602 (2001). arXiv:hep-ph/0106295

49. J.L. Feng, A.D. Shapere, Phys. Rev. Lett. 88, 021303 (2002). arXiv:hep-ph/0109106

50. A. Ringwald, H. Tu, Phys. Lett. B 525, 135 (2002). arXiv:hep-ph/0111042

51. M. Cavaglia, S. Das, R. Maartens, Class. Quantum Gravity 20, L205 (2003). arXiv:hep-ph/0305223

52. G. Dvali, M. Redi, Phys. Rev. D 77, 045027 (2008). arXiv:0710.4344

53. M. Casals, S. Dolan, P. Kanti, E. Winstanley, JHEP 06, 071 (2008). arXiv:0801.4910

54. G.L. Alberghi, L. Bellagamba, X. Calmet, R. Casadio, O. Micu, Eur. Phys. J. C 73, 2448 (2013). arXiv:1412.6719

55. P.F. Valeri, Z. Andrei. arXiv:1412.6719 [hep-th]

56. S. Hossenfelder, M. Bleicher, S. Hofmann, J. Ruppert, S. Scherer, H. Stocker, Phys. Lett. B 575, 85 (2003). arXiv:hep-th/0305262

57. S.I. Kruglov, Int. J. Mod. Phys. A 29, 1450118 (2014). arXiv: 1408.6561

58. F. Tangherlini, Nuovo Cim. 27, 636 (1963). doi:10.1007/ BF02784569

59. D.Y. Chen, H.W. Wu, H.T. Yang, S.Z. Yang, Int. J. Mod. Phys. A 29, 1430054 (2014)

60. E.T. Akhmedov, V. Akhmedova, D. Singleton, Phys. Lett. B 642, 124 (2006). arXiv:hep-th/0608098

61. E.T. Akhmedov, V. Akhmedova, D. Singleton, T. Pilling, Int. J. Mod. Phys. A 22, 1705 (2007). arXiv:hep-th/0605137

62. V. Akhmedova, T. Pilling, A. de Gill, D. Singleton, Phys. Lett. B 666, 269 (2008). arXiv:0804.2289

63. E.T. Akhmedov, T. Pilling, D. Singleton, Int. J. Mod. Phys. D 17, 2453 (2008). arXiv:0805.2653

64. B.D. Chowdhury, Pramana 70, 3 (2008). arXiv:hep-th/0605197

65. V. Akhmedova, T. Pilling, A. de Gill, D. Singleton, Phys. Lett. B 673, 227 (2009). arXiv:0808.3413

66. G. Amelino-Camelia, M. Arzano, A. Procaccini, Phys. Rev. D 70, 107501 (2004). arXiv:gr-qc/0405084

67. P. Nicolini. arXiv:1202.2102 [hep-th]

68. A.F. Ali, Phys. Rev. D 89, 104040 (2014). arXiv:1402.5320

69. A.F. Ali, M. Faizal, M.M. Khalil, JHEP 12, 159 (2014). arXiv: 1409.5745

70. A.F. Ali, M. Faizal, M.M. Khalil, Nucl. Phys. B 894, 341 (2015). arXiv: 1410.5706

71. A.H. Nima, D. Savas, D. Gia, Phys. Lett. B 429, 263 (1998). arXiv:hep-ph/9803315

72. C.M.S. Collaboration, JHEP 09, 094 (2012). arXiv:1206.5663

73. A.F. Ali, M. Faizal, M.M. Khalil, Phys. Lett. B 743, 295 (2015). arXiv: 1410.4765

74. ATLAS Collaboration, Phys. Lett. B 754, 302 (2016). arXiv: 1512.01530

75. J. Magueijo, L. Smolin, Phys. Rev. D 71, 026010 (2005). arXiv:hep-th/0401087

76. C. Bambi, F.R. Urban, Class. Quantum Gravity 25, 095006 (2008). arXiv:0709.1965

77. A.F. Ali, S. Das, E.C. Vagenas, Phys. Lett. B 678, 497 (2009). arXiv:0906.5396

78. P. Chen, Y.C. Ong, D.H. Yeom, JHEP 12, 021 (2014). arXiv: 1408.3763

79. P. Chen, Y.C. Ong, D.H. Yeom, Phys. Rep. 603, 1 (2015). arXiv: 1412.8366 